\title{
镍催化下一溴二氟甲烷对(杂)芳基溴代物的二氟甲基化反应
}

\author{
高兴何旭张新刚* \\ (中国科学院上海有机化学研究所 分子合成卓越中心 中国科学院有机氟化学重点实验室 上海 200032)
}

\begin{abstract}
摘要 二氟甲基取代的(杂)芳烃化合物由于二氟甲基的独特性质越来越受到了化学家和药物学家的广泛关注. 在过去 的几年中, 大量合成该类化合物的方法不断被发展，但这些方法中大多使用了价格昂贵的需要多步合成的二氟甲基化 试剂，从而制约了其广泛应用. 因此，发展以廉价易得的二氟甲基化试剂为氟源制备二氟甲基(杂)芳烃化合物的方法是 十分必要的. 以廉价易得的澳二氟甲烷 $\left(\mathrm{BrCF}_{2} \mathrm{H}\right)$ 为二氟甲基化试剂, 发展了镍催化下(杂)芳基溴代物与 $\mathrm{BrCF}_{2} \mathrm{H}$ 的偶联 反应. 该反应高效温和, 底物普适性好, 官能团兼容性优秀, 并且可以克量级合成, 为合成二氟甲基(杂)芳烃化合物提 供了一种高效简洁、廉价的方法. 初步机理研究表明, 该反应经历了一种镍催化的还原偶联历程.
\end{abstract}

关键词 芳基溴代物; 一溴二氟甲烷; 二氟甲基化; 镍; 还原偶联

\section{Nickel-Catalyzed Difluoromethylation of (Hetero)aryl Bromides with $\mathrm{BrCF}_{2} \mathrm{H}$}

\author{
Gao, Xing $\mathrm{He}, \mathrm{Xu}$ Zhang, Xingang* \\ (Key Laboratory of Organofluorine Chemistry, Center for Excellence in Molecular Synthesis, Shanghai Institute of Organic \\ Chemistry, Chinese Academy of Sciences, Shanghai 200032)
}

\begin{abstract}
A nickel-catalyzed direct difluoromethylation of (hetero)aryl bromides with bromodifluoromethane $\left(\mathrm{BrCF}_{2} \mathrm{H}\right)$ is described. This reaction features high efficiency, broad substrate scope and high functional group tolerance, providing a cost-efficient and straightforward route for applications in medicinal chemistry. Preliminary mechanistic studies reveal that a nickel-based, reductive cross-coupling catalytic cycle is involved in the reaction.
\end{abstract}

Keywords aryl bromide; bromodifluoromethane; difluoromethylation; nickel; reductive cross-coupling

\section{Introduction}

Difluoromethylated (hetero)arenes have gained increasing attention because of the unique properties of difluoromethyl group $\left(\mathrm{CF}_{2} \mathrm{H}\right)$ that can function as a lipophilic hydrogen bond donor, and also as a bioisostere of hydroxyl and thiol groups. ${ }^{[1]}$ Hence, it is of great interest to develop new and efficient methods to synthesize difluoromethylated (hetero)arenes. Transition-metal-catalyzed direct difluromethylation of (hetero)aromatics has emerged as one of the most efficient strategies to access difluoromethylated (hetero)arenes. ${ }^{[2]}$ However, most of the difluoromethylation reagents used in these reported methods are expensive and additional step(s) are required to synthesize from fluoroalkyl halides, ${ }^{[2 c, 3]}$ thus limiting their widespread applications.
In this regard, recently, we developed a palladium-catalyzed difluoromethylation of arylboronic acids with abundant and inexpensive industrial material chlorodifluoromethane $\left(\mathrm{ClCF}_{2} \mathrm{H}\right)$ via a metal-difluorocarbene coupling $(\mathrm{MeDiC}){ }^{[4]}$ which represents the first example of catalytic difluoromethylation from $\mathrm{ClCF}_{2} \mathrm{H}^{[5]}$ Later on, to replace the palladium with a base metal as a catalyst, we ${ }^{[6]}$ developed a nickel-catalyzed reductive cross-coupling between $\mathrm{ClCF}_{2} \mathrm{H}$ and (hetero)aryl chlorides/bromides. Despite of the importance of these methods, the developing new methods to access valuable difluoromethylated (hetero)arenes remain highly desirable. Bromodifluoromethane $\left(\mathrm{BrCF}_{2} \mathrm{H}\right)$ is a simple and readily available difluoromethyl source. ${ }^{[7]}$ Very recently, we ${ }^{[8]}$ and others ${ }^{[9]}$ reported a nickel-catalyzed

\footnotetext{
* Corresponding author. E-mail: xgzhang@sioc.ac.cn

Received August 15, 2018; revised September 14, 2018; published online October 14, 2018.

Dedicated to Professor Qingyun Chen on the occasion of his 90th birthday.

Project supported by the National Basic Research Program of China (973 Program, No. 2015CB931900), the National Natural Science Foundation of China (Nos. 21425208, 21672238, 21332010, 21421002), and the Strategic Priority Research Program of the Chinese Academy of Sciences (No. XDB20000000). 国家重点基础研究发展计划(973 计划, No. 2015CB931900)、国家自然科学基金(Nos. 21425208, 21672238, 21332010, 21421002)和中国科学院先导专项 (No. XDB20000000)资助项目.
} 
cross-coupling of $\mathrm{BrCF}_{2} \mathrm{H}$ with arylboronic acids, independently. However, the nickel-catalyzed reductive cross-coupling of $\mathrm{BrCF}_{2} \mathrm{H}$ with (hetero)aryl halides has not been reported so far. To continue our research on catalytic fluoroalkylation reactions with inexpensive and readily available fluoroalkyl halides as fluoroalkyl sources, ${ }^{[10]}$ herein, we disclose a nickel-catalyzed reductive crosscoupling of widely accessible (hetero)aryl bromides with $\mathrm{BrCF}_{2} \mathrm{H}$. The reaction proceeds under mild reaction conditions with broad substrate scope and excellent functional group tolerance without preformation of arylmetals.

\section{Results and discussion}

Initially, 4-bromo-1,1'-biphenyl (2a) was chosen as a coupling partner for this nickel-catalyzed difluoromethylation reaction (Table 1). However, only $16 \%$ yield of $3 \mathbf{a}$ was provided when the reaction was carried out with $\mathrm{BrCF}_{2} \mathrm{H}$ ( $1,1.0$ equiv.) and $2 \mathrm{a}$ (2.5 equiv.) in the presence of $\mathrm{NiBr}_{2} \cdot$ Diglyme (5 mol\%) and bypyridyl (bpy) (5 mol\%) in 1,3-dimethyl-3,4,5,6-tetrahydro-2( $1 H)$-pyrimidinone (DMPU) at $80{ }^{\circ} \mathrm{C}$ by employing $\mathrm{Zn}$ as a reductant (Entry 1). The low yield of $\mathbf{3 a}$ is because of the formation of hydrodebrominated compound $\mathrm{CF}_{2} \mathrm{H}_{2}(4,10 \%$ yield) and some other uncertain defluorinated by-products. To suppress these side reactions, a series of reaction parameters,

Table 1 Representative results for optimization of Ni-catalyzed difluoromethylation of $\mathbf{2 a}$ with $\mathrm{BrCF}_{2} \mathrm{H}(\mathbf{1})^{a}$

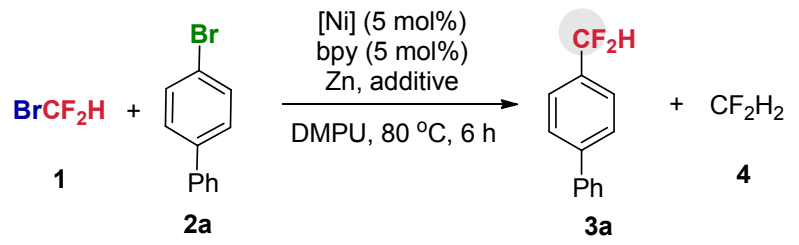

\begin{tabular}{|c|c|c|c|c|}
\hline Entry & {$[\mathrm{Ni}]$} & $\begin{array}{c}\mathrm{Zn} / \\
\text { equiv. }\end{array}$ & $\begin{array}{c}\text { Additive } \\
\text { (equiv.) }\end{array}$ & $\begin{array}{c}\text { Yield }^{b} / \% \text { of } \\
\mathbf{3 a} / \mathbf{4} / \mathbf{1} \\
\end{array}$ \\
\hline 1 & $\mathrm{NiBr}_{2} \cdot$ Diglyme & 1.2 & - & $16 / 10 / 36$ \\
\hline 2 & $\mathrm{NiBr}_{2} \cdot$ Diglyme & 1.2 & $\operatorname{LiI}(0.2)$ & 24/Trace/44 \\
\hline 3 & $\mathrm{NiBr}_{2} \bullet$ Diglyme & 1.2 & $\mathrm{NaI}(0.2)$ & $30 / 12 / 21$ \\
\hline 4 & $\mathrm{NiBr}_{2} \cdot$ Diglyme & 1.2 & $\mathrm{KI}(0.2)$ & $45 /$ Trace $/ 25$ \\
\hline 5 & $\mathrm{NiBr}_{2} \bullet$ Diglyme & 2.0 & $\mathrm{KI}(0.2)$ & $62 /$ Trace $/ 7$ \\
\hline 6 & $\mathrm{NiBr}_{2} \cdot$ Diglyme & 2.0 & KI (0.4) & $66 / 5 / 0$ \\
\hline 7 & $\mathrm{Ni}\left(\mathrm{PPh}_{3}\right)_{2} \mathrm{Br}_{2}$ & 2.0 & KI (0.4) & $73 / 5 / 0$ \\
\hline 8 & $\mathrm{Ni}\left(\mathrm{PPh}_{3}\right)_{2} \mathrm{Cl}_{2}$ & 2.0 & KI (0.4) & $60 / 12 / 0$ \\
\hline 9 & $\mathrm{Ni}($ dppe $) \mathrm{Cl}_{2}$ & 2.0 & KI (0.4) & $27 / 12 / 0$ \\
\hline 10 & $\mathrm{NiCl}_{2} \cdot \mathrm{DME}$ & 2.0 & $\mathrm{KI}(0.4)$ & 19/Trace/34 \\
\hline 11 & $\mathrm{Ni}(\operatorname{cod})_{2}$ & 2.0 & $\mathrm{KI}(0.4)$ & $0 / 8 / 45$ \\
\hline $12^{c}$ & $\mathrm{Ni}\left(\mathrm{PPh}_{3}\right)_{2} \mathrm{Br}_{2}$ & 2.0 & KI (0.4) & $78 / 4 / 0$ \\
\hline $13^{c, d}$ & $\mathrm{Ni}\left(\mathrm{PPh}_{3}\right)_{2} \mathrm{Br}_{2}$ & 2.0 & KI (0.4) & $90(91) /$ Trace $/ 0$ \\
\hline $14^{c, d}$ & - & 2.0 & KI (0.4) & $0 / 9 / 0$ \\
\hline $15^{c, d, e}$ & $\mathrm{Ni}\left(\mathrm{PPh}_{3}\right)_{2} \mathrm{Br}_{2}$ & 2.0 & KI (0.4) & $0 / 8 / 10$ \\
\hline
\end{tabular}

${ }^{a}$ Reaction conditions (unless otherwise specified): 1 ( $0.3 \mathrm{mmol}, 1.0$ equiv.), 2a ( $0.75 \mathrm{mmol}, 2.5$ equiv.), DMPU $(2 \mathrm{~mL}), 6 \mathrm{~h} .{ }^{b}$ Determined by ${ }^{19} \mathrm{~F}$ NMR using fluorobenzene as an internal standard and number in parenthesis is isolated yield. ${ }^{c} \mathrm{Ni}\left(\mathrm{PPh}_{3}\right)_{2} \mathrm{Br}_{2}(10 \mathrm{~mol} \%)$, bpy $(10 \mathrm{~mol} \%), 60{ }^{\circ} \mathrm{C}, 6 \mathrm{~h} .{ }^{d} \mathbf{1}(0.3 \mathrm{mmol}$, 1.0 equiv.), $2 \mathrm{a}\left(0.45 \mathrm{mmol}, 1.5\right.$ equiv.), DMPU $(1.5 \mathrm{~mL}) .{ }^{e}$ The reaction was run in the absence of bpy. such as ligand, solvent, and additive were examined (Entries $2 \sim 7$ ). Among the tested ligands and solvents, the bpy ligand and DMPU were still the best choice. Other diamine ligands, such as 1,10-phenanthroline (phen), 4,4'-di'Bu-bpy, even the electron-rich ligand 4,4'-diMeO-bpy, all provided lower yields. To our delight, the use of KI (0.4 equiv. $)^{[11]}$ as an additive could afford $\mathbf{3 a}$ in $66 \%$ yield albeit formation of $5 \%$ yield of $\mathrm{CF}_{2} \mathrm{H}_{2}$ (Entry 6). The reaction was sensitive to the nickel catalysts (Entries $7 \sim 11$ ) and $\mathrm{Ni}\left(\mathrm{PPh}_{3}\right)_{2} \mathrm{Br}_{2}$ showed the highest reactivity (Entry 6). Finally, the optimum reaction conditions were identified by decreasing the reaction temperature to $60{ }^{\circ} \mathrm{C}$ with utility of 1.5 equiv. of 2a and $10 \mathrm{~mol} \% \mathrm{Ni}\left(\mathrm{PPh}_{3}\right)_{2} \mathrm{Br}_{2} / \mathrm{bpy}$, providing compound 3a in $91 \%$ yield upon isolation (Entry 13). No product 3a was observed without nickel or bpy, thus demonstrating the essential role of Ni/bpy in promotion of the reaction (Entries 14 and 15).

With the viable reaction conditions in hand, a variety of aryl bromides were examined (Table 2). Generally, arylbromides bearing both electron-donating and electron-withdrawing substituents all showed good reactivity towards $\mathrm{BrCF}_{2} \mathrm{H}$, providing the corresponding difluoromethylated arenes in good to high yields $(\mathbf{3 c} \sim 3 \mathbf{3 i})$. The reaction showed high functional group compatibility. Many important functional groups, including alkoxycarbonyl, enolizable ketone silyl and cyano are compatible with the reaction conditions ( $(\mathbf{f} \sim \mathbf{3 k}$ ), even towards alcohol (3I) and arylboronate $(\mathbf{3 m})$, thus demonstrating the advantage of present nickel-catalyzed process. Importantly, heteroaryl bromides were also amenable to the reaction. Pyridine-, quinoline-, isoquinoline-, benzothiazole-, and benzooxazole-containing substrates all underwent the reaction smoothly, leading to the corresponding difluoromethylated heteroarenes in good yields $(\mathbf{3 p} \sim \mathbf{3 w})$. Gram-scale synthesis of difluoromethylated quinoline $3 \mathbf{t}$ also proceeded efficiently with comparable yield (64\%), thus demonstrating the reliability and practicability of this method. Furthermore, the utility of this method can also be demonstrated by the late-stage difluoromethylation of estrone- and ibuprofen-derived aryl bromides ( $\mathbf{3} \mathbf{x}$ and $\mathbf{3 y}$ ), providing potential opportunities for discovering new interesting bioactive molecules.

To gain some mechanistic insights into the current nickel-catalyzed process, several experiments were conducted. Firstly, the reaction of $\mathrm{BrCF}_{2} \mathrm{H}$ with $\mathrm{Zn}$ in DMPU was performed at $60{ }^{\circ} \mathrm{C}$ (Scheme 1a). It was found that the difluoromethyl zinc species $\left[\mathrm{BrZnCF}_{2} \mathrm{H}\right](\mathbf{A 1})$ and $\left[\mathrm{Zn}\left(\mathrm{CF}_{2} \mathrm{H}\right)_{2}\right](\mathbf{A 2})$ could be generated in a high yield $(\mathbf{A 1}+$ $\mathbf{A 2}=90 \%$ yield determined by ${ }^{19} \mathrm{~F}$ NMR ${ }^{[12]}$ under this reaction condition. However, no desired product $\mathbf{3 a}$ was observed when the mixture of $\mathbf{A 1}$ and $\mathbf{A 2}$ was treated with aryl bromide 2a in the presence of $\mathrm{Ni}\left(\mathrm{PPh}_{3}\right)_{2} \mathrm{Br}_{2}$ and bpy (Scheme 1a). In addition, the use of ${ }^{19} \mathrm{~F}$ NMR to monitor the reaction showed that $\mathbf{A 1}$ and $\mathbf{A 2}$ were not generated under standard reaction conditions (Scheme 1b). Thus, these results rule out the pathway that the production of difluoro- 
Table 2 Ni-catalyzed reductive cross-coupling of bromodifluoromethane (1) with aryl bromides (2) ${ }^{a}$

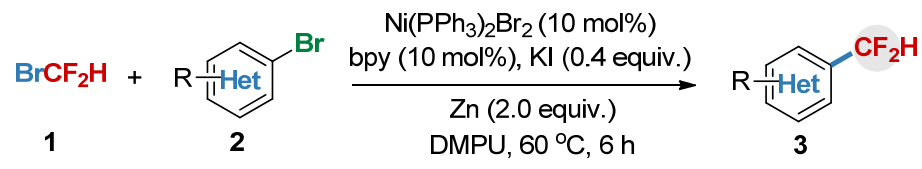

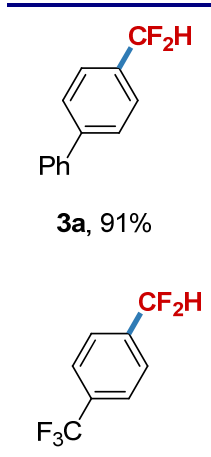

3i, $77 \%^{b}$

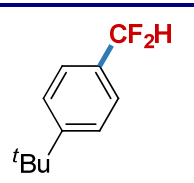

$3 \mathbf{b}, 74 \%^{b}$

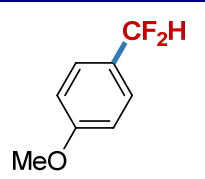

$3 c, 55 \%{ }^{b}$

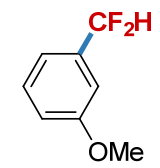

3d, $83 \%^{b}$

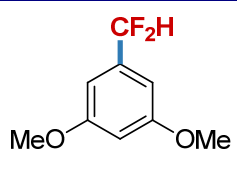

$3 e, 56 \%$

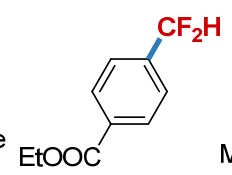

3f, $95 \%$

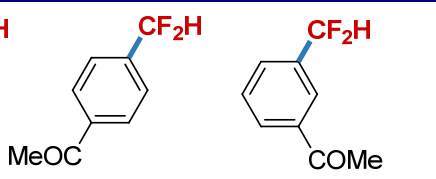

3g, $92 \%$

3h, $80 \%$

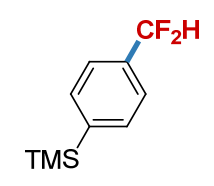

3j, $75 \%$

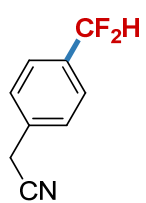

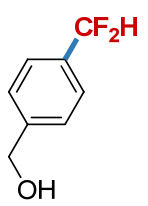

3I, $60 \%$

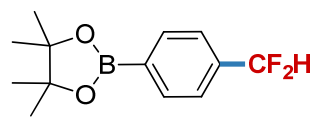

$3 \mathrm{~m}, 71 \%$<smiles>FC(F)(F)c1ccc2ccccc2c1</smiles>

3n, $60 \%$

3o, $84 \%$

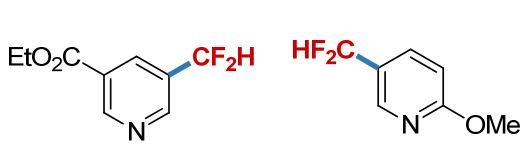

3p, $67 \%^{c}$

$3 q, 67 \%$,<smiles>FC(F)c1ccc(OCc2ccccc2)nc1</smiles>

$3 r, 47 \%^{c}$<smiles>Cc1ccc2cc(C(F)(F)F)ccc2n1</smiles>

3s, $62 \%$ 3t, $66 \%$ $64 \%^{d}$<smiles>FC(F)c1ccc2cnccc2c1</smiles>

$3 u, 59 \%$<smiles></smiles>

3v, $\mathrm{X}=\mathrm{S}, 74 \%^{c}$

$3 w, X=0,63 \%^{c}$

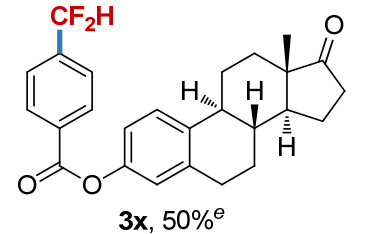<smiles>CC(C)Cc1ccc(C(C)C(=O)OCc2ccc(C(F)(F)F)cc2)cc1</smiles>

$3 y, 62 \%^{e}$

${ }^{a}$ Reaction conditions (unless otherwise specified): $2\left(0.9\right.$ mmol, 1.5 equiv.), 1 (0.6 mmol, 1.0 equiv.), DMPU $(3.0 \mathrm{~mL}), 60{ }^{\circ} \mathrm{C}, 6 \mathrm{~h}$. Yields of isolated products are given. ${ }^{b} \mathrm{NMR}$ yield determined by ${ }^{19} \mathrm{~F}$ NMR. ${ }^{c} 2$ (2.0 equiv.), 1 (0.6 mmol, 1.0 equiv.), Ni( $\left.\mathrm{PPh}_{3}\right) \mathrm{Br}_{2}(5 \mathrm{~mol} \%), 4,4^{\prime}-\mathrm{di}{ }^{i} \mathrm{Bu}$-bpy $(5 \mathrm{~mol} \%), 3 \AA \mathrm{MS}(200 \mathrm{mg}), 12 \mathrm{~h}$. ${ }^{d}$ Gram-scale synthesis and reaction run for $12 \mathrm{~h} .{ }^{e} \mathbf{2}$ (1.5 equiv.), 6 (0.3 mmol, 1.0 equiv.), $\mathrm{Ni}\left(\mathrm{PPh}_{3}\right) \mathrm{Br}_{2}(5 \mathrm{~mol} \%), 4,4^{\prime}$-dit $\mathrm{Bu}-\mathrm{bpy}(5 \mathrm{~mol} \%), 3 \AA \mathrm{MS}(400 \mathrm{mg}), 12 \mathrm{~h}$.
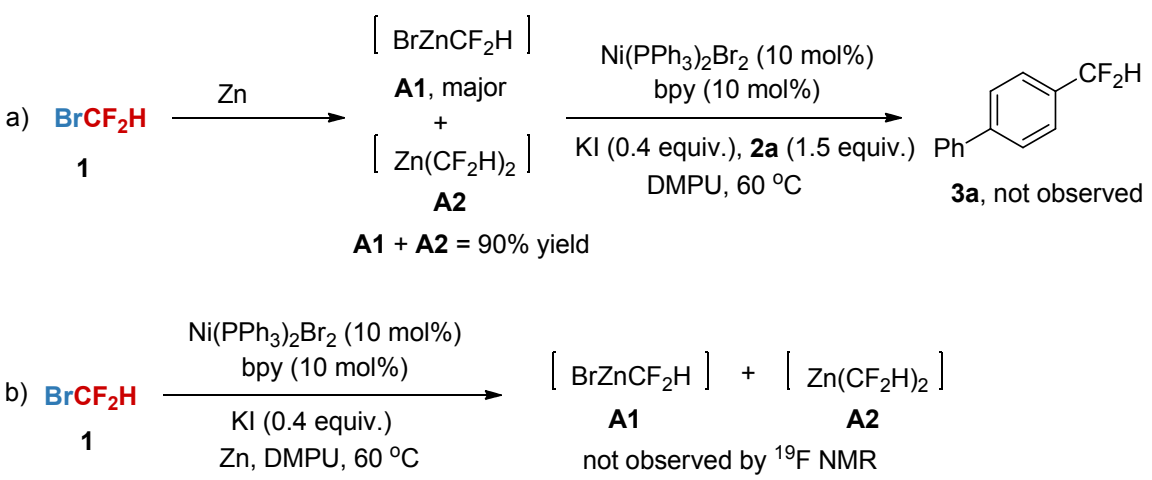

c)

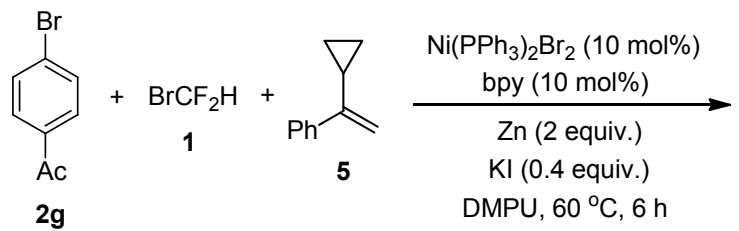

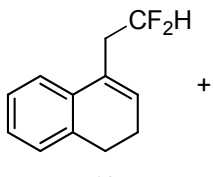

6, $14 \%$

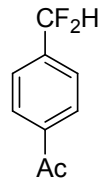

3g, $80 \%$

Scheme 1 Mechanistic studies 
methylated arenes is originated from the cross-coupling of difluoroalkyl zinc species with aryl bromides. Secondly, the radical clock experiment by employing $\alpha$-cyclopropylstyrene $\mathbf{5}$ as a probe ${ }^{[6]}$ showed that a ring-expanded product 6 was obtained in $14 \%$ yield, demonstrating that a difluoromethyl radical exists in the reaction (Scheme 1c).

On the basis of these results and previous reports, ${ }^{[6,13]}$ two possible pathways were proposed for the current nickel-catalyzed process (Scheme 2). Path I: The reaction begins the reaction of $\mathrm{Ni}(0)$ with aryl bromide to generate $\mathrm{Ni}(\mathrm{II})$ complex [( $\left.\mathrm{Ar}) \mathrm{Ni}^{\mathrm{II}}\left(\mathrm{L}_{\mathrm{n}}\right) \mathrm{Br}\right](\mathbf{A})$, which is subsequently reduced by $\mathrm{Zn}$ to give $\mathrm{Ni}(\mathrm{I})$ complex $\left[(\mathrm{Ar}) \mathrm{Ni}^{\mathrm{I}}\left(\mathrm{L}_{\mathrm{n}}\right)\right]$ (B) (Scheme $2 \mathrm{a}$ ). $\mathbf{B}$ reacts with $\mathrm{BrCF}_{2} \mathrm{H}$ via a radical cage rebound process to produce the key intermediate [(Ar) $\mathrm{Ni}^{\mathrm{iII}}$ $\left.\left(\mathrm{L}_{\mathrm{n}}\right)\left(\mathrm{CF}_{2} \mathrm{H}\right) \mathrm{Br}\right](\mathbf{C})$. Finally, $\mathbf{C}$ undergoes reductive elimination to provide the difluoromethylated arene and $\left[\operatorname{BrNi}^{\mathrm{I}}\left(\mathrm{L}_{\mathrm{n}}\right)\right]$ (D). Reduction of $\mathbf{D}$ by $\mathrm{Zn}$ regenerates the $\left[\mathrm{Ni}^{0}\left(\mathrm{~L}_{\mathrm{n}}\right)\right]$ species. Path II: The difluoromethyl radical generated between $\left[\mathrm{BrNi}^{\mathrm{I}}\left(\mathrm{L}_{n}\right)\right](\mathbf{D})$ and $\mathrm{BrCF}_{2} \mathrm{H}$ diffuses to the solution to react with $\mathbf{A}$ to give the key intermediate $\mathbf{C}$ (Scheme 2b). Subsequently, reductive elimination of $\mathbf{C}$ provides the difluoromethylated arene. Finally, the resulting nickel(II) complex $\left[\mathrm{Ni}^{\mathrm{II}}\left(\mathrm{L}_{n}\right) \mathrm{Br}_{2}\right]$ is reduced by $\mathrm{Zn}$ to regenerate $\left[\mathrm{Ni}^{0}\left(\mathrm{~L}_{n}\right)\right]$.

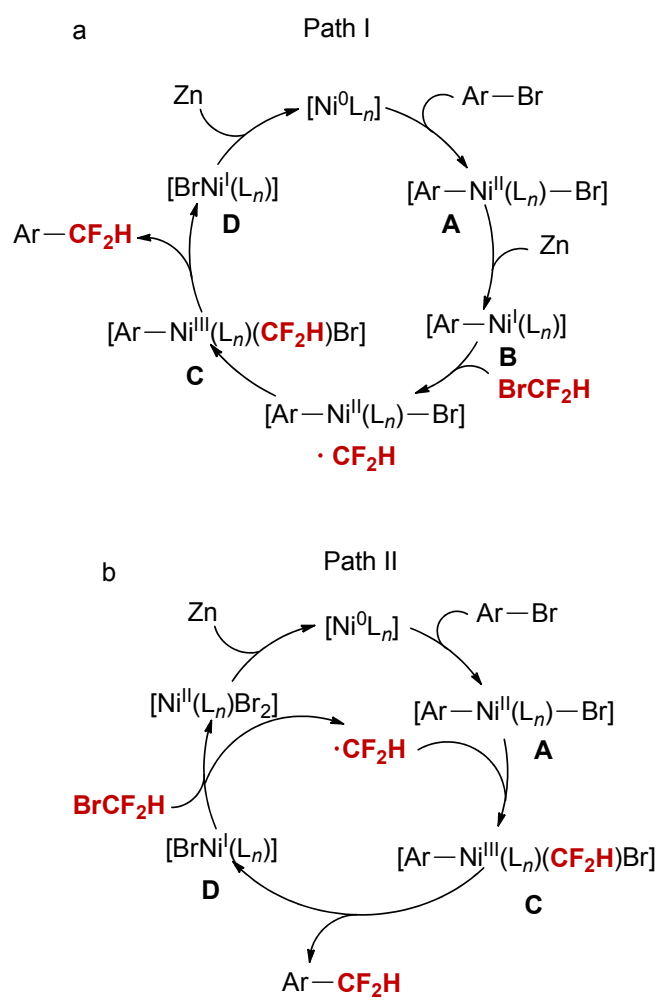

Scheme 2 Proposed reaction mechanisms

\section{Conclusions}

In conclusion, we have developed a nickel-catalyzed direct difluoromethylation of (hetero)aryl bromides with simple and readily available $\mathrm{BrCF}_{2} \mathrm{H}$ via a reductive cross-coupling. The reaction proceeds under mild reaction conditions with high efficiency and good functional groups tolerance, providing a cost-efficient and straightforward route for the synthesis of difluoromethylated (hetero)arenes that are of great interest in medicinal chemistry.

\section{Experimental section}

\subsection{General information}

${ }^{1} \mathrm{H}$ NMR and ${ }^{13} \mathrm{C}$ NMR spectra were recorded on an Agilent MR 400 and Agilent MR 500 spectrometer. ${ }^{19} \mathrm{~F}$ NMR was recorded on an Agilent MR 400 spectrometer $\left(\mathrm{CFCl}_{3}\right.$ as an external standard and low field is positive). NMR yield was determined by ${ }^{19} \mathrm{~F}$ NMR using fluorobenzene as an internal standard before working up the reaction.

All reagents were used as received from commercial sources, unless otherwise stated, or prepared as described in the literature. 1,3-dimethyl-3,4,5,6-tetrahydro-2( $1 H)$-pyrimidinone (DMPU) was purchased from TCI and distilled from calcium hydride for twice before use.

\subsection{Preparation of $\mathrm{BrCF}_{2} \mathrm{H}$ stock solution}

1,4-Dioxane $(15 \mathrm{~mL})$ was added to a Schlenk tube under argon. $\mathrm{BrCF}_{2} \mathrm{H}$ gas was then bubbled through the 1,4-dioxane until the total volume of the solution reach the maximum. The concentration of the $\mathrm{BrCF}_{2} \mathrm{H}\left(3 \mathrm{~mol} \cdot \mathrm{L}^{-1}\right)$ stock solution was determined by ${ }^{19} \mathrm{~F}$ NMR spectrum using fluorobenzene as an internal standard.

\subsection{General procedure for Ni-catalyzed cross-coup-} ling of 1 with 2

To a $25 \mathrm{~mL}$ of Schlenk tube were added (hetero)arylbromide 2 ( 0.9 mmol, 1.5 equiv.), $\mathrm{Ni}\left(\mathrm{PPh}_{3}\right)_{2} \mathrm{Br}_{2}(10$ $\mathrm{mol} \%$ ), bpy (10 mol\%), KI (0.4 equiv.), and $\mathrm{Zn}$ (2.0 equiv.) under air. The mixture was then evacuated and backfilled with argon for three times. DMPU ( $3 \mathrm{~mL})$ and $\mathrm{BrCF}_{2} \mathrm{H}(2.5$ $\mathrm{mol} \cdot \mathrm{L}^{-1}$ in 1,4 -dioxane, $240 \mu \mathrm{L}, 0.6 \mathrm{mmol}, 1.0$ equiv.) were added subsequently. The Schlenk tube was screw capped and put into a preheated oil bath $\left(60{ }^{\circ} \mathrm{C}\right)$. After stirring for 6 $\mathrm{h}$, the reaction mixture was cooled to room temperature, diluted with ethyl acetate, and filtered through a pad of celite. The filtrate was concentrated, and the residue was washed with $10 \mathrm{~mL}$ of water, and extracted with organic solvent $[V($ petroleum ether $): V($ EtOAc $)=20: 1]$ for three times. The combined organic layers were washed with brine, dried over $\mathrm{Na}_{2} \mathrm{SO}_{4}$ and concentrated. The residue was purified with silica gel chromatography to give product 3 .

4-(Difluoromethyl)-1,1'-biphenyl (3a): ${ }^{[4]}$ The product (111 mg, 91\% yield) was purified with silica gel chromatography (petroleum ether) as a white solid. ${ }^{1} \mathrm{H}$ NMR (400 $\left.\mathrm{MHz}, \mathrm{CDCl}_{3}\right) \delta: 7.72 \sim 7.68(\mathrm{~m}, 2 \mathrm{H}), 7.64 \sim 7.59(\mathrm{~m}, 4 \mathrm{H})$, $7.52 \sim 7.47(\mathrm{~m}, 2 \mathrm{H}), 7.44 \sim 7.49(\mathrm{~m}, 1 \mathrm{H}), 6.71(\mathrm{t}, J=56.4$ $\mathrm{Hz}, 1 \mathrm{H}) ;{ }^{19} \mathrm{~F}$ NMR $\left(376 \mathrm{MHz}, \mathrm{CDCl}_{3}\right) \delta:-110.3(\mathrm{~d}, J=$ $56.5 \mathrm{~Hz}, 2 \mathrm{~F}) ;{ }^{13} \mathrm{C} \mathrm{NMR}\left(125.7 \mathrm{MHz}, \mathrm{CDCl}_{3}\right) \delta: 143.7(\mathrm{t}$, $J=2.0 \mathrm{~Hz}), 140.2,133.2(\mathrm{t}, J=22.4 \mathrm{~Hz}), 128.9,127.9$, 127.4, 127.2, $126.0(\mathrm{t}, J=6.0 \mathrm{~Hz}), 114.7$ (t, $J=238.5 \mathrm{~Hz})$.

1-(tert-Butyl)-4-(difluoromethyl)benzene $(\mathbf{3 b}):^{[4]}$ The product (74\% yield determined by ${ }^{19} \mathrm{~F}$ NMR) was purified with silica gel chromatography (petroleum ether) as a col- 
orless oil. ${ }^{1} \mathrm{H}$ NMR (400 MHz, $\left.\mathrm{CDCl}\right) \delta: 7.50 \sim 7.44(\mathrm{~m}$, $4 \mathrm{H}), 6.63(\mathrm{t}, J=56.8 \mathrm{~Hz}, 1 \mathrm{H}), 1.35(\mathrm{~s}, 9 \mathrm{H}) ;{ }^{19} \mathrm{~F}$ NMR $(376$ $\left.\mathrm{MHz}, \mathrm{CDCl}_{3}\right) \delta$ : $-109.9(\mathrm{~d}, J=56.6 \mathrm{~Hz}, 2 \mathrm{~F}) ;{ }^{13} \mathrm{C} \mathrm{NMR}$ $\left(101 \mathrm{MHz}, \mathrm{CDCl}_{3}\right) \delta: 154.0(\mathrm{t}, J=2.0 \mathrm{~Hz}), 131.5(\mathrm{t}, J=22.4$ $\mathrm{Hz}), 125.6,125.3$ (t, $J=6.0 \mathrm{~Hz}), 114.9$ (t, $J=237.9 \mathrm{~Hz})$, 34.8, 31.2 .

1-(Difluoromethyl)-4-methoxybenzene (3c): $:^{[4]}$ The product (55\% yield determined by ${ }^{19} \mathrm{~F} \mathrm{NMR}$ ) as colorless oil was purified with silica gel chromatography [V(petroleum ether $): V($ EtOAc $)=100: 1) .{ }^{1} \mathrm{H} \mathrm{NMR}\left(400 \mathrm{MHz}, \mathrm{CDCl}_{3}\right)$ $\delta: 7.44(\mathrm{~d}, J=8.6 \mathrm{~Hz}, 2 \mathrm{H}), 6.96(\mathrm{~d}, J=8.8 \mathrm{~Hz}, 2 \mathrm{H}), 6.60(\mathrm{t}$, $J=56.7 \mathrm{~Hz}, 1 \mathrm{H}), 3.84(\mathrm{~s}, 3 \mathrm{H}) ;{ }^{19} \mathrm{~F}$ NMR $\left(376 \mathrm{MHz}, \mathrm{CDCl}_{3}\right)$ $\delta:-108.3(\mathrm{~d}, J=56.8 \mathrm{~Hz}, 2 \mathrm{~F}) ;{ }^{13} \mathrm{C}$ NMR $(125.7 \mathrm{MHz}$, $\left.\mathrm{CDCl}_{3}\right) \delta: 161.33,127.1(\mathrm{t}, J=6.0 \mathrm{~Hz}), 126.74(\mathrm{t}, J=22.7$ $\mathrm{Hz}), 114.9$ (t, $J=237.3 \mathrm{~Hz}), 114.0,55.3$.

1-(Difluoromethyl)-3-methoxybenzene (3d): ${ }^{[4]}$ The product ( $83 \%$ yield, determined by ${ }^{19} \mathrm{~F}$ NMR) was characterized by ${ }^{19} \mathrm{~F}$ NMR and GC-MS analysis. ${ }^{19} \mathrm{~F}$ NMR (376 $\left.\mathrm{MHz}, \mathrm{CDCl}_{3}\right) \delta:-111.3(\mathrm{~d}, J=37.6 \mathrm{~Hz}, 2 \mathrm{~F})$; GC-MS $m / z$ (\%): $158.0\left(\mathrm{M}^{+}\right)$.

1-(Difluoromethyl)-3, 5-dimethoxybenzene (3e): ${ }^{[4]}$ The product $(63 \mathrm{mg}, 56 \%$ yield) was purified with silica gel chromatography $[V($ petroleum ether $): V($ EtOAc $)=30: 1]$ as a colorless oil. ${ }^{1} \mathrm{H} \mathrm{NMR}\left(400 \mathrm{MHz}, \mathrm{CDCl}_{3}\right) \delta: 6.68 \sim$ $6.62(\mathrm{~m}, 2 \mathrm{H}), 6.56(\mathrm{t}, J=56.4 \mathrm{~Hz}, 1 \mathrm{H}), 6.56 \sim 6.52(\mathrm{~m}, 1 \mathrm{H})$, $3.82(\mathrm{~s}, 6 \mathrm{H}) ;{ }^{19} \mathrm{~F}$ NMR $\left(376 \mathrm{MHz}, \mathrm{CDCl}_{3}\right) \delta:-110.9(\mathrm{~d}$, $J=56.4 \mathrm{~Hz}, 2 \mathrm{~F}) ;{ }^{13} \mathrm{C} \mathrm{NMR}\left(101 \mathrm{MHz}, \mathrm{CDCl}_{3}\right) \delta: 161.0$, $136.3(\mathrm{t}, J=22.4 \mathrm{~Hz}), 114.5(\mathrm{t}, J=239.4 \mathrm{~Hz}), 103.4(\mathrm{t}, J=$ $6.3 \mathrm{~Hz}), 102.6(\mathrm{t}, J=1.8 \mathrm{~Hz}), 55.5$.

Ethyl 4-(difluoromethyl)benzoate (3f): ${ }^{[4]}$ The product (114 mg, 95\% yield) was purified with silica gel chromatography $[V$ (petroleum ether) $: V($ EtOAc $)=10: 1]$ as a colorless oil. ${ }^{1} \mathrm{H}$ NMR $\left(400 \mathrm{MHz}, \mathrm{CDCl}_{3}\right) \delta: 8.13$ (d, $J=8.2$ $\mathrm{Hz}, 2 \mathrm{H}), 7.58$ (d, $J=8.1 \mathrm{~Hz}, 2 \mathrm{H}), 6.69$ (t, $J=56.1 \mathrm{~Hz}, 1 \mathrm{H})$, 4.40 (q, $J=7.1 \mathrm{~Hz}, 2 \mathrm{H}), 1.41(\mathrm{t}, J=7.1 \mathrm{~Hz}, 3 \mathrm{H}) ;{ }^{19} \mathrm{~F}$ NMR $\left(376 \mathrm{MHz}, \mathrm{CDCl}_{3}\right) \delta:-112.2(\mathrm{~d}, J=56.1 \mathrm{~Hz}, 2 \mathrm{~F}) ;{ }^{13} \mathrm{C}$ NMR (101 MHz, $\left.\mathrm{CDCl}_{3}\right) \delta: 165.7,138.3(\mathrm{t}, J=22.4 \mathrm{~Hz})$, 132.7 (t, $J=1.8 \mathrm{~Hz}), 129.9,125.6(\mathrm{t}, J=6.1 \mathrm{~Hz}), 114.0$ (t, $J=239.7 \mathrm{~Hz}), 61.3,14.3$.

1-(4-(Difluoromethyl)phenyl)ethanone $\quad(\mathbf{3 g}):^{[4]} \quad$ The product (94 mg, 92\% yield) was purified with silica gel chromatography $[V($ petroleum ether $): V($ EtOAc $)=10: 1]$ as a colorless oil. ${ }^{1} \mathrm{H}$ NMR $\left(400 \mathrm{MHz}, \mathrm{CDCl}_{3}\right) \delta: 8.04(\mathrm{~d}$, $J=8.0 \mathrm{~Hz}, 2 \mathrm{H}), 7.61(\mathrm{~d}, J=7.9 \mathrm{~Hz}, 2 \mathrm{H}), 6.69$ (t, $J=56.1$ $\mathrm{Hz}, 1 \mathrm{H}), 2.64(\mathrm{~s}, 3 \mathrm{H}) ;{ }^{19} \mathrm{~F}$ NMR $\left(376 \mathrm{MHz}, \mathrm{CDCl}_{3}\right) \delta$ : $-112.3(\mathrm{~d}, J=56.1 \mathrm{~Hz}, 2 \mathrm{~F}) ;{ }^{13} \mathrm{C} \mathrm{NMR}\left(101 \mathrm{MHz}, \mathrm{CDCl}_{3}\right)$ $\delta: 197.3,138.8(\mathrm{t}, J=1.8 \mathrm{~Hz}), 138.5(\mathrm{t}, J=22.4 \mathrm{~Hz}), 128.6$, $125.9(\mathrm{t}, J=6.0 \mathrm{~Hz}), 113.9(\mathrm{t}, J=239.8 \mathrm{~Hz}), 26.8$.

1-(3-(Difluoromethyl)phenyl)ethanone $\quad(\mathbf{3 h}):^{[4]}$ The product (82 $\mathrm{mg}, 80 \%$ yield) was purified with silica gel chromatography $[V($ petroleum ether $): V($ EtOAc $)=10: 1]$ as a colorless oil. ${ }^{1} \mathrm{H}$ NMR $\left(400 \mathrm{MHz}, \mathrm{CDCl}_{3}\right) \delta: 8.06(\mathrm{~s}$, $1 \mathrm{H}), 8.04$ (d, $J=8.2 \mathrm{~Hz}, 1 \mathrm{H}), 7.69$ (d, $J=7.7 \mathrm{~Hz}, 1 \mathrm{H}), 7.55$ $(\mathrm{t}, J=7.7 \mathrm{~Hz}, 1 \mathrm{H}), 6.68(\mathrm{t}, J=56.2 \mathrm{~Hz}, 1 \mathrm{H}), 2.61(\mathrm{~s}, 3 \mathrm{H})$; ${ }^{19} \mathrm{~F}$ NMR $\left(376 \mathrm{MHz}, \mathrm{CDCl}_{3}\right) \delta:-111.2(\mathrm{~d}, J=56.2 \mathrm{~Hz}$, $2 \mathrm{~F}) ;{ }^{13} \mathrm{C} \mathrm{NMR}\left(101 \mathrm{MHz}, \mathrm{CDCl}_{3}\right) \delta: 197.0,137.4,134.8(\mathrm{t}$, $J=22.8 \mathrm{~Hz}), 130.4$ (t, $J=1.7 \mathrm{~Hz}), 129.9$ (t, $J=5.8 \mathrm{~Hz})$,
129.1, 125.4 (t, $J=6.2 \mathrm{~Hz}), 114.1$ (t, $J=239.4 \mathrm{~Hz}), 26.5$.

1-(Difluoromethyl)-4-(trifluoromethyl)benzene $\quad(3 \mathbf{i}):^{[4]}$ The product (77\% yield, determined by ${ }^{19} \mathrm{~F}$ NMR) was characterized by ${ }^{19} \mathrm{~F}$ NMR and GC-MS analysis. ${ }^{19} \mathrm{~F}$ NMR $\left(376 \mathrm{MHz}, \mathrm{CDCl}_{3}\right) \delta:-113.6(\mathrm{~d}, J=37.6 \mathrm{~Hz}, 2 \mathrm{~F}),-64.0$ (s, 3F); GC-MS m/z (\%): $196.0\left(\mathrm{M}^{+}\right)$.

(4-(Difluoromethyl)phenyl)trimethylsilane $(\mathbf{3 j}):^{[4]}$ The product $(90 \mathrm{mg}, 75 \%$ yield) was purified with silica gel chromatography (petroleum ether) as a colorless oil. ${ }^{1} \mathrm{H}$ NMR (400 MHz, $\left.\mathrm{CDCl}_{3}\right) \delta: 7.61(\mathrm{~d}, J=8.1 \mathrm{~Hz}, 2 \mathrm{H}), 7.48$ $(\mathrm{d}, J=7.8 \mathrm{~Hz}, 2 \mathrm{H}), 6.63(\mathrm{t}, J=56.4 \mathrm{~Hz}, 1 \mathrm{H}), 0.28$ (s, 9H); ${ }^{19} \mathrm{~F}$ NMR $\left(376 \mathrm{MHz}, \mathrm{CDCl}_{3}\right) \delta$ : $-110.9(\mathrm{~d}, J=56.5 \mathrm{~Hz}$, $2 \mathrm{~F}) ;{ }^{13} \mathrm{C}$ NMR $\left(101 \mathrm{MHz}, \mathrm{CDCl}_{3}\right) \delta: 144.0(\mathrm{t}, J=1.7 \mathrm{~Hz})$, $134.6(\mathrm{t}, J=22.1 \mathrm{~Hz}), 133.6,124.7(\mathrm{t}, J=6.0 \mathrm{~Hz}), 114.8(\mathrm{t}$, $J=238.5 \mathrm{~Hz}),-1.3$.

2-(4-(Difluoromethyl)phenyl)acetonitrile (3k): The product $(73 \mathrm{mg}, 73 \%$ yield) was purified with silica gel chromatography $[V$ (petroleum ether) $: V($ EtOAc $)=20: 1]$ as a colorless oil. ${ }^{1} \mathrm{H} \mathrm{NMR}\left(400 \mathrm{MHz}, \mathrm{CDCl}_{3}\right) \delta$ : $7.52(\mathrm{~d}$, $J=8.0 \mathrm{~Hz}, 2 \mathrm{H}), 7.42(\mathrm{~d}, J=8.0 \mathrm{~Hz}, 2 \mathrm{H}), 6.65$ (t, $J=56.3$ $\mathrm{Hz}, 1 \mathrm{H}), 3.79(\mathrm{~s}, 2 \mathrm{H}) ;{ }^{19} \mathrm{~F}$ NMR $\left(376 \mathrm{MHz}, \mathrm{CDCl}_{3}\right) \delta$ : $-111.0(\mathrm{~d}, J=56.3 \mathrm{~Hz}, 2 \mathrm{~F}) ;{ }^{13} \mathrm{C}$ NMR $\left(101 \mathrm{MHz}, \mathrm{CDCl}_{3}\right)$ $\delta: 134.2(\mathrm{t}, J=22.7 \mathrm{~Hz}), 132.6(\mathrm{t}, J=2.0 \mathrm{~Hz}), 128.2,126.3$ (t, $J=6.1 \mathrm{~Hz}), 117.3,114.1(\mathrm{t}, J=238.9 \mathrm{~Hz}), 23.4$; IR (film) $v_{\max }: 2965,2253,1621,1425,1328 \mathrm{~cm}^{-1}$; MS (EI) $m / z(\%)$ : $167\left(\mathrm{M}^{+}\right), 116$ (100); HRMS calcd for $\mathrm{C}_{9} \mathrm{H}_{7} \mathrm{NF}_{2}$ 167.0547, found 167.0552 .

(4-(Difluoromethyl)phenyl)methanol (31): ${ }^{[4]}$ The product (57 mg, 60\% yield) was purified with silica gel chromatography $[V$ (petroleum ether) $: V($ EtOAc $)=10: 1]$ as a colorless oil. ${ }^{1} \mathrm{H}$ NMR (400 MHz, $\left.\mathrm{CDCl}_{3}\right) \delta: 7.48$ (d, $J=8.1$ $\mathrm{Hz}, 2 \mathrm{H}), 7.41(\mathrm{~d}, J=8.0 \mathrm{~Hz}, 2 \mathrm{H}), 6.63(\mathrm{t}, J=56.5 \mathrm{~Hz}, 1 \mathrm{H})$, $4.69(\mathrm{~s}, 2 \mathrm{H}), 2.48(\mathrm{~s}, 1 \mathrm{H}) ;{ }^{19} \mathrm{~F}$ NMR $\left(376 \mathrm{MHz}, \mathrm{C}_{6} \mathrm{D}_{6}\right) \delta$ : $-111.6(\mathrm{~d}, J=56.5 \mathrm{~Hz}, 2 \mathrm{~F}) ;{ }^{13} \mathrm{C}$ NMR $\left(101 \mathrm{MHz}, \mathrm{CDCl}_{3}\right)$ $\delta: 143.5(\mathrm{t}, J=1.9 \mathrm{~Hz}), 133.5(\mathrm{t}, J=22.4 \mathrm{~Hz}), 126.9,125.7$ (t, $J=6.1 \mathrm{~Hz}), 114.6(\mathrm{t}, J=238.4 \mathrm{~Hz}), 64.5$.

2-(4-(Difluoromethyl)phenyl)-4,4,5,5-tetramethyl-1,3,2dioxaborolane (3m): The product (108 $\mathrm{mg}, 71 \%$ yield) was purified with silica gel chromatography $[V($ petroleum ether) $: V($ EtOAc $)=100: 1]$ as a colorless oil. ${ }^{1} \mathrm{H}$ NMR $\left(400 \mathrm{MHz}, \mathrm{CDCl}_{3}\right) \delta: 7.89(\mathrm{~d}, J=7.7 \mathrm{~Hz}, 2 \mathrm{H}), 7.50(\mathrm{~d}, J=$ $7.8 \mathrm{~Hz}, 2 \mathrm{H}), 6.65(\mathrm{t}, J=56.4 \mathrm{~Hz}, 1 \mathrm{H}), 1.35(\mathrm{~s}, 12 \mathrm{H}) ;{ }^{19} \mathrm{~F}$ NMR (282 MHz, $\left.\mathrm{CDCl}_{3}\right) \delta:-111.9(\mathrm{~d}, J=56.4 \mathrm{~Hz}, 2 \mathrm{~F})$; ${ }^{13} \mathrm{C}$ NMR (101 MHz, $\left.\mathrm{CDCl}_{3}\right) \delta: 136.8(\mathrm{t}, J=22.1 \mathrm{~Hz})$, 135.0, 126.0 (t, $J=6.1 \mathrm{~Hz}), 124.7(\mathrm{t}, J=6.0 \mathrm{~Hz}), 114.7$ (t, $J=239.0 \mathrm{~Hz}$ ), 84.1, 24.9; IR (film) $v_{\max }: 2984,1742,1374$ $\mathrm{cm}^{-1}$; MS (EI) $m / z(\%): 254\left(\mathrm{M}^{+}\right), 239$ (100); HRMS calcd. for $\mathrm{C}_{13} \mathrm{H}_{16}{ }^{10} \mathrm{BO}_{2} \mathrm{~F}_{2}\left(\mathrm{M}-\mathrm{H}^{+}\right)$252.1248, found 252.1247.

1-(Difluoromethyl)naphthalene (3n): ${ }^{[4]}$ The product (64 $\mathrm{mg}, 60 \%$ yield) was purified with silica gel chromatography (Petroleum ether) as a colorless oil. ${ }^{1} \mathrm{H}$ NMR $(300 \mathrm{MHz}$, $\left.\mathrm{CDCl}_{3}\right) \delta: 8.20(\mathrm{~d}, J=8.0 \mathrm{~Hz}, 1 \mathrm{H}), 7.98(\mathrm{~d}, J=8.3 \mathrm{~Hz}, 1 \mathrm{H})$, $7.93(\mathrm{~d}, J=8.0 \mathrm{~Hz}, 1 \mathrm{H}), 7.71(\mathrm{~d}, J=7.1 \mathrm{~Hz}, 1 \mathrm{H}), 7.67 \sim$ $7.44(\mathrm{~m}, 3 \mathrm{H}), 7.15(\mathrm{t}, J=55.1 \mathrm{~Hz}, 1 \mathrm{H}) ;{ }^{19} \mathrm{~F} \mathrm{NMR}(376 \mathrm{MHz}$, $\left.\mathrm{CDCl}_{3}\right) \delta:-110.9(\mathrm{~d}, J=55.1 \mathrm{~Hz}, 2 \mathrm{~F}) ;{ }^{13} \mathrm{C}$ NMR $(101$ $\left.\mathrm{MHz}, \mathrm{CDCl}_{3}\right) \delta: 133.7,131.5(\mathrm{t}, J=1.8 \mathrm{~Hz}), 129.7(\mathrm{t}, J=$ $2.9 \mathrm{~Hz}), 129.5$ (t, $J=20.9 \mathrm{~Hz}), 128.7,127.1,126.3,124.8(\mathrm{t}$, 
$J=8.7 \mathrm{~Hz}), 124.6,123.5(\mathrm{t}, J=1.4 \mathrm{~Hz}), 115.4(\mathrm{t}, J=238.3$ $\mathrm{Hz})$.

2-(Difluoromethyl)naphthalene (3o): ${ }^{[4]}$ The product $(85$ $\mathrm{mg}, 84 \%$ yield) was purified with silica gel chromatography (petroleum ether) as a colorless oil. ${ }^{1} \mathrm{H}$ NMR $(400 \mathrm{MHz}$, $\left.\mathrm{CDCl}_{3}\right) \delta: 7.98(\mathrm{~s}, 1 \mathrm{H}), 7.96 \sim 7.85(\mathrm{~m}, 3 \mathrm{H}), 7.66 \sim 7.50(\mathrm{~m}$, $3 \mathrm{H}), 6.81(\mathrm{t}, J=56.4 \mathrm{~Hz}, 1 \mathrm{H}) ;{ }^{19} \mathrm{~F}$ NMR $\left(376 \mathrm{MHz}, \mathrm{CDCl}_{3}\right)$ $\delta$ : $-109.9(\mathrm{~d}, J=56.4 \mathrm{~Hz}, 2 \mathrm{~F}) ;{ }^{13} \mathrm{C}$ NMR $(101 \mathrm{MHz}$, $\left.\mathrm{CDCl}_{3}\right) \delta: 134.3(\mathrm{t}, J=1.4 \mathrm{~Hz}), 132.5,131.6(\mathrm{t}, J=22.2$ $\mathrm{Hz}), 128.9,128.5,127.9,127.4,126.8,125.9$ (t, $J=7.5 \mathrm{~Hz}$ ), $122.0(\mathrm{t}, J=4.8 \mathrm{~Hz}), 115.0(\mathrm{t}, J=238.5 \mathrm{~Hz})$.

Ethyl 5-(difluoromethyl)nicotinate $(\mathbf{3 p}):^{[14]}$ The reaction was carried out with ethyl 5-bromonicotinate (2.0 equiv), 1 (0.6 mmol, 1.0 equiv), $\mathrm{Ni}\left(\mathrm{PPh}_{3}\right) \mathrm{Br}_{2}$ (5 mol\%), 4,4'-dit $\mathrm{Bu}-$ bpy (5 mol\%), and molecular sieves ( $3 \AA, 200 \mathrm{mg})$ in DMPU $(3 \mathrm{~mL})$ at $60{ }^{\circ} \mathrm{C}$ for $12 \mathrm{~h}$. The product $(81 \mathrm{mg}, 67 \%$ yield) was purified with silica gel chromatography $[V($ petroleum ether $): V($ EtOAc $)=8: 1]$ as a colorless oil. ${ }^{1} \mathrm{H}$ NMR (500 MHz, $\left.\mathrm{CDCl}_{3}\right) \delta: 9.31(\mathrm{~s}, 1 \mathrm{H}), 8.90(\mathrm{~s}, 1 \mathrm{H})$, $8.42(\mathrm{~s}, 1 \mathrm{H}), 6.76(\mathrm{t}, J=55.6 \mathrm{~Hz}, 1 \mathrm{H}), 4.43(\mathrm{q}, J=7.1 \mathrm{~Hz}$, $2 \mathrm{H}), 1.41(\mathrm{t}, J=7.1 \mathrm{~Hz}, 3 \mathrm{H}) ;{ }^{19} \mathrm{~F}$ NMR $\left(376 \mathrm{MHz}, \mathrm{CDCl}_{3}\right)$ $\delta:-112.9(\mathrm{~d}, J=55.6 \mathrm{~Hz}, 2 \mathrm{~F}) ;{ }^{13} \mathrm{C}$ NMR $(125.7 \mathrm{MHz}$, $\left.\mathrm{CDCl}_{3}\right) \delta: 164.3,152.8,150.5(\mathrm{t}, J=6.4 \mathrm{~Hz}), 134.4(\mathrm{t}, J=$ $5.7 \mathrm{~Hz}), 130.0(\mathrm{t}, J=23.5 \mathrm{~Hz}), 126.4,112.8(\mathrm{t}, J=240.3$ $\mathrm{Hz}), 61.9$, 14.2; IR (film) $v_{\max }$ : 3434, 2986, 1728, 1293, 1214, $1027 \mathrm{~cm}^{-1}$; MS (EI) $\mathrm{m} / z(\%): 201\left(\mathrm{M}^{+}\right), 156(100)$; HRMS calcd for $\mathrm{C}_{9} \mathrm{H}_{9} \mathrm{NO}_{2} \mathrm{~F}_{2}\left(\mathrm{M}^{+}\right)$201.0601, found 201.0605 .

5-(Difluoromethyl)-2-methoxypyridine $(\mathbf{3 q}):^{[6]}$ The reaction was carried out with 5-bromo-2-methoxypyridine (2.0 equiv.), 1 ( 0.6 mmol, 1.0 equiv.), $\mathrm{Ni}\left(\mathrm{PPh}_{3}\right) \mathrm{Br}_{2}$ (5 mol\%), 4,4'-di'Bu-bpy (5 mol\%), and molecular sieves (3 $\AA, 200 \mathrm{mg})$ in DMPU $(3 \mathrm{~mL})$ at $60{ }^{\circ} \mathrm{C}$ for $12 \mathrm{~h}$. The product (67\% yield, determined by ${ }^{19} \mathrm{~F}$ NMR) was characterized by ${ }^{19} \mathrm{~F}$ NMR and GC-MS analysis. ${ }^{19} \mathrm{~F}$ NMR (376 MHz, $\left.\mathrm{CDCl}_{3}\right) \delta$ : $-109.6(\mathrm{~d}, J=56.4 \mathrm{~Hz}, 2 \mathrm{~F})$; GC-MS $m / z(\%)$ : $159.0\left(\mathrm{M}^{+}\right), 158(100), 140,129$.

2-(Benzyloxy)-5-(difluoromethyl)pyridine $(3 \mathbf{3 r}):^{[5]}$ The reaction was carried out with 2-benzyloxy-5-bromopyridine (2.0 equiv.), 1 ( $0.6 \mathrm{mmol}, 1.0$ equiv.), $\mathrm{Ni}\left(\mathrm{PPh}_{3}\right) \mathrm{Br}_{2}$ (5 mol\%), 4,4'-di'Bu-bpy (5 mol\%), and molecular sieves (3 $\AA, 200 \mathrm{mg})$ in DMPU $(3 \mathrm{~mL})$ at $60{ }^{\circ} \mathrm{C}$ for $12 \mathrm{~h}$. The product (66 mg, 47\% yield) was purified with silica gel chromatography $[V$ (petroleum ether $): V($ EtOAc $)=10: 1]$ as a colorless oil. ${ }^{1} \mathrm{H}$ NMR $\left(400 \mathrm{MHz}, \mathrm{CDCl}_{3}\right) \delta: 8.30$ (d, $J=1.6$ $\mathrm{Hz}, 1 \mathrm{H}), 7.74$ (dd, $J=8.6 \mathrm{~Hz}, 2.3 \mathrm{~Hz}, 1 \mathrm{H}), 7.46$ (d, $J=7.1$ $\mathrm{Hz}, 2 \mathrm{H}), 7.43 \sim 7.29(\mathrm{~m}, 3 \mathrm{H}), 6.88(\mathrm{~d}, J=8.6 \mathrm{~Hz}, 1 \mathrm{H}), 6.65$ $(\mathrm{t}, J=56.0 \mathrm{~Hz}, 1 \mathrm{H}), 5.42(\mathrm{~s}, 2 \mathrm{H}) ;{ }^{19} \mathrm{~F} \mathrm{NMR}(376 \mathrm{MHz}$, $\left.\mathrm{CDCl}_{3}\right) \delta:-109.7(\mathrm{~d}, J=56.4 \mathrm{~Hz}, 2 \mathrm{~F}) ;{ }^{13} \mathrm{C}$ NMR $(126$ $\left.\mathrm{MHz}, \mathrm{CDCl}_{3}\right) \delta: 165.1(\mathrm{t}, J=1.3 \mathrm{~Hz}), 145.2(\mathrm{t}, J=7.5 \mathrm{~Hz})$, $136.8,135.9(\mathrm{t}, J=4.5 \mathrm{~Hz}), 128.5,128.0,128.0,123.6(\mathrm{t}$, $J=23.4 \mathrm{~Hz}$ ), 113.8 (t, $J=237.6 \mathrm{~Hz}), 111.7,68.1$; IR (film) $v_{\max }: 2956,1614,1500,1349,1288,1081,1017 \mathrm{~cm}^{-1}$; MS (EI) $m / z(\%): 235\left(\mathrm{M}^{+}\right), 91(100)$; HRMS calcd. for $\mathrm{C}_{13} \mathrm{H}_{11} \mathrm{NOF}_{2}$ 235.0809, found 201.0802.

3-(Difluoromethyl)quinoline (3s): ${ }^{[14]}$ The product (67 $\mathrm{mg}, 62 \%$ yield) was purified with silica gel chromatography
$[V($ petroleum ether $): V($ EtOAc $)=8: 1]$ as a colorless oil. ${ }^{1} \mathrm{H}$ NMR $\left(500 \mathrm{MHz}, \mathrm{CDCl}_{3}\right) \delta: 9.03(\mathrm{~d}, J=1.9 \mathrm{~Hz}, 1 \mathrm{H})$, 8.29 (s, 1H), $8.16(\mathrm{~d}, J=8.5 \mathrm{~Hz}, 1 \mathrm{H}), 7.89$ (d, $J=8.2 \mathrm{~Hz}$, $1 \mathrm{H}), 7.83 \sim 7.78(\mathrm{~m}, 1 \mathrm{H}), 7.65 \sim 7.60(\mathrm{~m}, 1 \mathrm{H}), 6.88(\mathrm{t}, J=$ $55.8 \mathrm{~Hz}, 1 \mathrm{H}) ;{ }^{19} \mathrm{~F}$ NMR $\left(376 \mathrm{MHz}, \mathrm{CDCl}_{3}\right) \delta$ : $-111.6(\mathrm{~d}$, $J=55.8 \mathrm{~Hz}, 2 \mathrm{~F}) .{ }^{13} \mathrm{C} \mathrm{NMR}\left(125.7 \mathrm{MHz}, \mathrm{CDCl}_{3}\right) \delta: 149.0(\mathrm{t}$, $J=1.5 \mathrm{~Hz}), 147.1(\mathrm{t}, J=5.3 \mathrm{~Hz}), 133.9(\mathrm{t}, J=6.7 \mathrm{~Hz})$, 131.0, 129.5, 128.3, 127.6, 127.1 (t, $J=22.8 \mathrm{~Hz}), 126.8$, $113.7(\mathrm{t}, J=239.5 \mathrm{~Hz})$; IR (film) $v_{\max }: 3420,3061,2964$, 1625, 1500, 1180, 1089, $1032 \mathrm{~cm}^{-1}$; MS (EI) $\mathrm{m} / \mathrm{z}(\%): 179$ $\left(\mathrm{M}^{+}\right)$, 179(100); HRMS calcd for $\mathrm{C}_{10} \mathrm{H}_{7} \mathrm{NF}_{2}$ 179.0547, found 179.0541 .

6-(Difluoromethyl)-2-methylquinoline (3t): ${ }^{[6]}$ The product (76 mg, 66\% yield) was purified with silica gel chromatography $[V$ (petroleum ether) $: V($ EtOAc $)=8: 1]$ as white solid (m.p. $\left.48 \sim 50{ }^{\circ} \mathrm{C}\right) .{ }^{1} \mathrm{H} \mathrm{NMR}\left(400 \mathrm{MHz}, \mathrm{CDCl}_{3}\right)$ $\delta: 8.10 \sim 8.01(\mathrm{~m}, 2 \mathrm{H}), 7.87(\mathrm{~s}, 1 \mathrm{H}), 7.76(\mathrm{~d}, J=8.8 \mathrm{~Hz}$, $1 \mathrm{H}), 7.31(\mathrm{~d}, J=8.4 \mathrm{~Hz}, 1 \mathrm{H}), 6.78(\mathrm{t}, J=56.3 \mathrm{~Hz}, 1 \mathrm{H}), 2.74$ $(\mathrm{s}, 3 \mathrm{H}) ;{ }^{19} \mathrm{~F}$ NMR $\left(376 \mathrm{MHz}, \mathrm{CDCl}_{3}\right) \delta:-110.2(\mathrm{~d}, J=$ $56.4 \mathrm{~Hz}, 2 \mathrm{~F}) ;{ }^{13} \mathrm{C}$ NMR $\left(125.7 \mathrm{MHz}, \mathrm{CDCl}_{3}\right) \delta: 160.7,148.6$ $(\mathrm{t}, J=1.5 \mathrm{~Hz}), 136.5,131.4(\mathrm{t}, J=22.5 \mathrm{~Hz}), 129.6,125.7(\mathrm{t}$, $J=4.9 \mathrm{~Hz}), 125.7,125.4(\mathrm{t}, J=7.2 \mathrm{~Hz}), 122.8,114.5(\mathrm{t}$, $J=238.9 \mathrm{~Hz}$ ), 25.4; IR (film) $v_{\max }: 2997,1631,1604,1484$, 1086, $1019 \mathrm{~cm}^{-1}$; MS (EI) m/z (\%): $193\left(\mathrm{M}^{+}\right), 193$ (100); HRMS calcd. for $\mathrm{C}_{11} \mathrm{H}_{9} \mathrm{NF}_{2} \quad 193.0703$, found 193.0695 .

6-(Difluoromethyl)isoquinoline (3u): The product (63 $\mathrm{mg}, 59 \%$ yield) was purified with silica gel chromatography $[V$ (petroleum ether) $: V($ EtOAc $)=8: 1]$ as a colorless oil. ${ }^{1} \mathrm{H}$ NMR $\left(500 \mathrm{MHz}, \mathrm{CDCl}_{3}\right) \delta: 9.29(\mathrm{~s}, 1 \mathrm{H}), 8.59(\mathrm{~d}, J=5.7$ $\mathrm{Hz}, 1 \mathrm{H}), 8.03$ (d, $J=8.5 \mathrm{~Hz}, 1 \mathrm{H}), 7.93(\mathrm{~s}, 1 \mathrm{H}), 7.68$ (t, $J=$ $6.6 \mathrm{~Hz}, 2 \mathrm{H}), 6.79(\mathrm{t}, J=56.0 \mathrm{~Hz}, 1 \mathrm{H}) ;{ }^{19} \mathrm{~F} \mathrm{NMR}(376 \mathrm{MHz}$, $\left.\mathrm{CDCl}_{3}\right) \delta:-111.7(\mathrm{~d}, J=56.4 \mathrm{~Hz}, 2 \mathrm{~F}) ;{ }^{13} \mathrm{C} \mathrm{NMR}(125.7$ $\left.\mathrm{MHz}, \mathrm{CDCl}_{3}\right) \delta: 152.4,143.9,135.9(\mathrm{t}, J=22.4 \mathrm{~Hz}), 135.1$, 129.1, 128.6, $124.3(\mathrm{t}, J=7.4 \mathrm{~Hz}), 123.5$ (t, $J=4.9 \mathrm{~Hz})$, $120.8,114.1\left(\mathrm{t}, J=239.8 \mathrm{~Hz}\right.$ ); IR (film) $v_{\max }: 3393,1591$, 1352, 1179, $1034 \mathrm{~cm}^{-1}$; MS (EI) $\mathrm{m} / z(\%): 179\left(\mathrm{M}^{+}\right), 179$ (100); HRMS calcd. for $\mathrm{C}_{10} \mathrm{H}_{7} \mathrm{NF}_{2}$ 179.0547, found 179.0545 .

2-(4-(Difluoromethyl)phenyl)benzo[ $[d]$ thiazole $(3 \mathbf{v})$ : The reaction was carried out with 1-(2-benzothiazolyl)-4-bromobenzene (2.0 equiv.), 1 ( $0.6 \mathrm{mmol}, 1.0$ equiv.), $\mathrm{Ni}\left(\mathrm{PPh}_{3}\right) \mathrm{Br}_{2}$ ( $5 \mathrm{~mol} \%$ ), 4,4'-di' $\mathrm{Bu}$-bpy ( $5 \mathrm{~mol} \%)$, and molecular sieves (3 $\AA, 200 \mathrm{mg})$ in DMPU $(3 \mathrm{~mL})$ at $60{ }^{\circ} \mathrm{C}$ for $12 \mathrm{~h}$. The product (116 mg, 74\% yield) was purified with silica gel chromatography $[V$ (petroleum ether) $: V($ EtOAc $)=5: 1]$ as a white solid. m.p. $103 \sim 105{ }^{\circ} \mathrm{C} ;{ }^{1} \mathrm{H}$ NMR $(400 \mathrm{MHz}$, $\left.\mathrm{CDCl}_{3}\right) \delta: 8.16(\mathrm{~d}, J=8.2 \mathrm{~Hz}, 2 \mathrm{H}), 8.09(\mathrm{~d}, J=8.1 \mathrm{~Hz}, 1 \mathrm{H})$, $7.90(\mathrm{~d}, J=8.0 \mathrm{~Hz}, 1 \mathrm{H}), 7.62(\mathrm{~d}, J=8.1 \mathrm{~Hz}, 2 \mathrm{H}), 7.57 \sim$ $7.46(\mathrm{~m}, 1 \mathrm{H}), 7.45 \sim 7.36(\mathrm{~m}, 1 \mathrm{H}), 6.70(\mathrm{t}, J=56.3 \mathrm{~Hz}, 1 \mathrm{H})$; ${ }^{19} \mathrm{~F}$ NMR $\left(376 \mathrm{MHz}, \mathrm{CDCl}_{3}\right) \delta$ : $-111.6(\mathrm{~d}, J=56.4 \mathrm{~Hz}$, $2 \mathrm{~F}) ;{ }^{13} \mathrm{C} \mathrm{NMR}\left(125.7 \mathrm{MHz}, \mathrm{CDCl}_{3}\right) \delta: 166.6,154.0,136.4$ (t, $J=22.5 \mathrm{~Hz}), 135.7$ (t, $J=2.0 \mathrm{~Hz}), 135.1,127.7,126.5$, $126.2(\mathrm{t}, J=6.1 \mathrm{~Hz}), 125.5,123.4,121.6,114.1(\mathrm{t}, J=239.4$ $\mathrm{Hz}$ ); IR (film) $v_{\max }: 3054,1484,1376,1073,1015 \mathrm{~cm}^{-1}$; MS (EI) $m / z(\%): 261\left(\mathrm{M}^{+}\right), 261$ (100); HRMS calcd for $\mathrm{C}_{14} \mathrm{H}_{9} \mathrm{NSF}_{2} 261.0424$, found 261.0422.

2-(4-(Difluoromethyl)phenyl)benzo[ $[d]$ oxazole $\quad(\mathbf{3 w}):^{[5]}$ 
The reaction was carried out with 2-(4-bromophenyl)-1,3benzoxazole ( 2.0 equiv.), 1 ( $0.6 \mathrm{mmol}, 1.0$ equiv.), $\mathrm{Ni}\left(\mathrm{PPh}_{3}\right)$ $\mathrm{Br}_{2}$ (5 mol\%), 4,4'-di' ${ }^{t} \mathrm{Bu}-$ bpy $(5 \mathrm{~mol} \%)$, and molecular sieves ( $3 \AA, 200 \mathrm{mg})$ in DMPU $(3 \mathrm{~mL})$ at $60{ }^{\circ} \mathrm{C}$ for $12 \mathrm{~h}$. The product (93 mg, 63\% yield) was purified with silica gel chromatography $[V($ petroleum ether $): V($ EtOAc $)=5: 1]$ as a white solid. m.p. $98 \sim 100{ }^{\circ} \mathrm{C} ;{ }^{1} \mathrm{H}$ NMR $(400 \mathrm{MHz}$, $\left.\mathrm{CDCl}_{3}\right) \delta: 8.33(\mathrm{~d}, J=8.1 \mathrm{~Hz}, 2 \mathrm{H}), 7.85 \sim 7.75(\mathrm{~m}, 1 \mathrm{H})$, $7.66(\mathrm{~d}, J=8.1 \mathrm{~Hz}, 2 \mathrm{H}), 7.62 \sim 7.55(\mathrm{~m}, 1 \mathrm{H}), 7.40 \sim 7.32$ $(\mathrm{m}, 2 \mathrm{H}), 6.71(\mathrm{t}, J=56.2 \mathrm{~Hz}, 1 \mathrm{H}) ;{ }^{19} \mathrm{~F}$ NMR $(376 \mathrm{MHz}$, $\left.\mathrm{CDCl}_{3}\right) \delta:-111.9(\mathrm{~d}, J=56.4 \mathrm{~Hz}, 2 \mathrm{~F}) ;{ }^{13} \mathrm{C}$ NMR $(101$ $\left.\mathrm{MHz}, \mathrm{CDCl}_{3}\right) \delta: 161.9(\mathrm{t}, J=1.0 \mathrm{~Hz}), 150.8,141.9,137.0(\mathrm{t}$, $J=22.5 \mathrm{~Hz}), 129.4(\mathrm{t}, J=2.0 \mathrm{~Hz}), 127.8,126.1(\mathrm{t}, J=6.1$ $\mathrm{Hz}), 125.5,124.8,120.2(\mathrm{~d}, J=1.0 \mathrm{~Hz}), 114.1(\mathrm{t}, J=239.6$ Hz), 110.7; IR (film) $v_{\max }: 3064,1557,1454,1073,1053$, $1015 \mathrm{~cm}^{-1}$; MS (EI) $\mathrm{m} / z(\%): 245\left(\mathrm{M}^{+}\right), 245$ (100); HRMS calcd for $\mathrm{C}_{14} \mathrm{H}_{9} \mathrm{NOF}_{2} 245.0652$, found 245.0653.

(13S)-13-Methyl-17-oxo-7,8,9,11,12,13,14,15,16,17decahydro- $6 H$-cyclopenta $[a]$ phenanthren-3-yl 4-(difluoromethyl)benzoate (3x): To a $25 \mathrm{~mL}$ Schlenk tube were added $2 \times\left(0.45 \mathrm{mmol}, 1.5\right.$ equiv.), $\mathrm{Ni}\left(\mathrm{PPh}_{3}\right)_{2} \mathrm{Br}_{2}$ (5 mol\%), $4,4^{\prime}$-di ${ }^{t}$ Bu-bpy (5 mol\%), zinc dust (2.0 equiv.), KI (0.4 equiv.) and $3 \AA \mathrm{MS}$ (400 mg). The mixture was evacuated and backfilled with argon for three times, then DMPU (1.5 $\mathrm{mL})$ and $\mathrm{BrCF}_{2} \mathrm{H} 1\left(2.5 \mathrm{~mol} \cdot \mathrm{L}^{-1}\right.$ in 1,4-dioxane, $0.3 \mathrm{mmol}$, 1.0 equiv.) were added subsequently. The Schlenk tube was screw capped and put into a preheated oil bath $\left(60{ }^{\circ} \mathrm{C}\right)$. After stirring overnight, the reaction mixture was cooled to room temperature and filtered with a pad of celite. The filtrate was concentrated, and the residue was purified with silica gel chromatography [ $V$ (petroleum ether) : $V$ (EtO$\mathrm{Ac})=58: 1]$ to give product $\mathbf{3 x}(64 \mathrm{mg}, 50 \%$ yield $)$ as a white solid. m.p. $140 \sim 143{ }^{\circ} \mathrm{C} ;{ }^{1} \mathrm{H}$ NMR $(400 \mathrm{MHz}$, $\left.\mathrm{CDCl}_{3}\right) \delta: 8.28(\mathrm{~d}, J=8.1 \mathrm{~Hz}, 2 \mathrm{H}), 7.66(\mathrm{~d}, J=8.1 \mathrm{~Hz}, 2 \mathrm{H})$, $7.35(\mathrm{~d}, \quad J=8.4 \mathrm{~Hz}, 1 \mathrm{H}), 6.99(\mathrm{~d}, J=8.4 \mathrm{~Hz}, 1 \mathrm{H}), 6.96$ (s, $1 \mathrm{H}), 6.73(\mathrm{t}, J=56.1 \mathrm{~Hz}, 1 \mathrm{H}), 2.99 \sim 2.92(\mathrm{~m}, 2 \mathrm{H}), 2.57 \sim$ $2.47(\mathrm{~m}, 1 \mathrm{H}), 2.47 \sim 2.40(\mathrm{~m}, 1 \mathrm{H}), 2.37 \sim 2.27(\mathrm{~m}, 1 \mathrm{H})$, $2.22 \sim 1.94(\mathrm{~m}, 4 \mathrm{H}), 1.71 \sim 1.42(\mathrm{~m}, 6 \mathrm{H}), 0.93(\mathrm{~s}, 3 \mathrm{H}) ;{ }^{19} \mathrm{~F}$ NMR (376 MHz, $\left.\mathrm{CDCl}_{3}\right) \delta:-112.4(\mathrm{~d}, J=56.4 \mathrm{~Hz}, 2 \mathrm{~F})$; ${ }^{13} \mathrm{C} \mathrm{NMR}\left(101 \mathrm{MHz}, \mathrm{CDCl}_{3}\right) \delta: 220.7,164.6,148.6,139.0$ (t, $J=22.5 \mathrm{~Hz}), 138.2,137.7,131.9,130.5,126.5,125.8$ (t, $J=6.1 \mathrm{~Hz}), 121.6,118.7,113.9(\mathrm{t}, J=239.9 \mathrm{~Hz}), 50.5,47.9$, 44.2, 38.0, 35.9, 31.6, 29.4, 26.3, 25.8, 21.6, 13.8; IR (film) $v_{\max }: 2934,2862,1734,1492,1270,1220,1067 \mathrm{~cm}^{-1}$; MS (EI) $m / z(\%): 424\left(\mathrm{M}^{+}\right), 155$ (100); HRMS calcd for $\mathrm{C}_{26} \mathrm{H}_{26} \mathrm{O}_{3} \mathrm{~F}_{2} 424.1850$, found 424.1855 .

4-(Difluoromethyl)benzyl 2-(4-isobutylphenyl) propanoate (3y): To a $25 \mathrm{~mL}$ Schlenk tube were added $2 \mathbf{y}(0.45$ mmol, 1.5 equiv. $), \mathrm{Ni}\left(\mathrm{PPh}_{3}\right)_{2} \mathrm{Br}_{2}(5 \mathrm{~mol} \%), 4,4^{\prime}-\mathrm{di}^{t} \mathrm{Bu}-$ bpy ( $5 \mathrm{~mol} \%$ ), zinc dust (2.0 equiv.), KI ( 0.4 equiv.) and $3 \AA$ MS (400 mg). The mixture was evacuated and backfilled with argon for three times, then DMPU $(1.5 \mathrm{~mL})$ and $\mathrm{BrCF}_{2} \mathrm{H} 1$ ( $2.5 \mathrm{~mol} \cdot \mathrm{L}^{-1}$ in 1,4 -dioxane, $0.3 \mathrm{mmol}, 1.0$ equiv.) were added subsequently. The Schlenk tube was screw capped and put into a preheated oil bath $\left(60{ }^{\circ} \mathrm{C}\right)$. After stirring overnight, the reaction mixture was cooled to room temperature and filtered with a pad of celite. The filtrate was concentrated, and the residue was purified with silica gel chromatography $[V($ petroleum ether $): V($ EtOAc $)=10: 1]$ to give product $3 \mathbf{y}\left(64 \mathrm{mg}, 62 \%\right.$ yield) as a colorless oil. ${ }^{1} \mathrm{H}$ NMR (400 MHz, $\left.\mathrm{CDCl}_{3}\right) \delta: 7.44(\mathrm{~d}, J=7.9 \mathrm{~Hz}, 2 \mathrm{H}), 7.29$ $(\mathrm{d}, J=8.0 \mathrm{~Hz}, 2 \mathrm{H}), 7.21$ (d, $J=8.0 \mathrm{~Hz}, 2 \mathrm{H}), 7.11(\mathrm{~d}, J=8.0$ $\mathrm{Hz}, 2 \mathrm{H}), 6.62(\mathrm{t}, J=56.4 \mathrm{~Hz}, 1 \mathrm{H}), 5.15(\mathrm{~s}, 2 \mathrm{H}), 3.78(\mathrm{q}$, $J=7.1 \mathrm{~Hz}, 1 \mathrm{H}), 2.47(\mathrm{~d}, J=7.2 \mathrm{~Hz}, 2 \mathrm{H}), 1.93 \sim 1.81(\mathrm{~m}$, $1 \mathrm{H}), 1.53(\mathrm{~d}, J=7.2 \mathrm{~Hz}, 3 \mathrm{H}), 0.92(\mathrm{~d}, J=6.6 \mathrm{~Hz}, 6 \mathrm{H}) ;{ }^{19} \mathrm{~F}$ NMR $\left(376 \mathrm{MHz}, \mathrm{CDCl}_{3}\right) \delta$ : $-110.7(\mathrm{~d}, J=56.4 \mathrm{~Hz}, 2 \mathrm{~F})$; ${ }^{13} \mathrm{C}$ NMR $\left(101 \mathrm{MHz}, \mathrm{CDCl}_{3}\right) \delta: 174.3,140.7,138.9(\mathrm{t}, J=$ $2.0 \mathrm{~Hz}), 137.4,134.0(\mathrm{t}, J=22.4 \mathrm{~Hz}), 129.3,127.7,127.2$, $125.7(\mathrm{t}, J=6.1 \mathrm{~Hz}), 114.5(\mathrm{t}, J=238.7 \mathrm{~Hz}), 65.5,45.1$, 45.0, 30.2, 22.3, 18.3; IR (film) $v_{\max }: 2956,2869,1735$, 1381, 1158, 1074, $1021 \mathrm{~cm}^{-1}$; MS (EI) $m / z(\%): 346\left(\mathrm{M}^{+}\right)$, 161 (100); HRMS calcd for $\mathrm{C}_{21} \mathrm{H}_{24} \mathrm{O}_{2} \mathrm{~F}_{2}$ 346.1744, found 346.1741 .

\subsection{Gram-scale synthesis of compound $3 t$}

To a $100 \mathrm{~mL}$ of Schlenk tube were added 6-bromo-2methylquinoline (13.5 mmol, 1.5 equiv.), $\mathrm{Ni}\left(\mathrm{PPh}_{3}\right)_{2} \mathrm{Br}_{2}(10$ $\mathrm{mol} \%)$, bpy (10 mol\%), zinc dust (2.0 equiv.) and KI (0.4 equiv.). The mixture was evacuated and backfilled with argon for three times, DMPU (45 mL) and $\mathrm{BrCF}_{2} \mathrm{H} 1$ (2.5 $\mathrm{mol} \cdot \mathrm{L}^{-1}$ in 1,4 -dioxane, $9 \mathrm{mmol}, 1.0$ equiv.) were added subsequently. The Schlenk tube was screw capped and put into a preheated oil bath $\left(60{ }^{\circ} \mathrm{C}\right)$. After stirring overnight, the reaction mixture was cooled to room temperature. The reaction mixture was filtered with a pad of celite. The filtrate was added into $50 \mathrm{~mL}$ of water. The aqueous layer was extracted with a mixture of petroleum ether and EtOAc $(V: V=5: 1 ; 100 \mathrm{~mL} \times 3)$. The combined organic layers were dried over anhydrous $\mathrm{Na}_{2} \mathrm{SO}_{4}$ and then evaporated under reduced pressure and the resulting crude product was purified by column chromatography with ethyl acetate and petroleum ether $(V: V=1: 5)$ as eluent to give product $3 \mathbf{t}$ $(1.12 \mathrm{~g}, 64 \%)$ as a white solid (m.p. $\left.48 \sim 50{ }^{\circ} \mathrm{C}\right)$.

\subsection{Radcial clock experiment}

To a $25 \mathrm{~mL}$ of Schlenk tube were added $\mathbf{2 g}(0.9 \mathrm{mmol}$, 1.5 equiv. $), \mathrm{Ni}\left(\mathrm{PPh}_{3}\right)_{2} \mathrm{Br}_{2}(10 \mathrm{~mol} \%)$, bpy $(10 \mathrm{~mol} \%), \mathrm{KI}$ ( 0.4 equiv.), and $\mathrm{Zn}$ dust (2.0 equiv.) under air. The mixture was then evacuated and backfilled with $\operatorname{Ar}$ (3 times). Compound 5 (0.9 mmol, 1.5 equiv.), DMPU ( $3 \mathrm{~mL}$ ) and $\mathrm{BrCF}_{2} \mathrm{H}\left(2.5 \mathrm{~mol} \cdot \mathrm{L}^{-1}\right.$ in dioxane, $240 \mu \mathrm{L}, 0.6 \mathrm{mmol}, 1.0$ equiv) were added subsequently. The Schlenk tube was screw capped and put into a preheated oil bath $\left(60{ }^{\circ} \mathrm{C}\right)$. After stirring for $6 \mathrm{~h}$, the reaction mixture was cooled to room temperature, diluted with ethyl acetate, and filtered through a pad of celite. The filtrate was concentrated, and the residue was washed with $10 \mathrm{~mL}$ of water, extracted with organic solvent $[V$ (petroleum ether $): V($ EtOAc $)=20: 1]$ for three times. The organic layer was washed with brine, dried over $\mathrm{Na}_{2} \mathrm{SO}_{4}$ and concentrated. The residue was purified by silica gel (petroleum ether) to give compound $\mathbf{6}^{[6]}$ (16 mg, 14\% yield). ${ }^{1} \mathrm{H}$ NMR (400 MHz, $\left.\mathrm{CDCl}_{3}\right) \delta: 7.22 \sim$ $7.11(\mathrm{~m}, 4 \mathrm{H}), 6.02(\mathrm{t}, J=4.4 \mathrm{~Hz}, 1 \mathrm{H}), 5.91(\mathrm{tt}, J=56.8,4.8$ $\mathrm{Hz}, 1 \mathrm{H}), 2.97(\mathrm{td}, J=16.5,3.8 \mathrm{~Hz}, 2 \mathrm{H}), 2.75(\mathrm{t}, J=8.1 \mathrm{~Hz}$, 2H), 2.28 (dd, $J=12.5,8.0 \mathrm{~Hz}, 2 \mathrm{H}) ;{ }^{19} \mathrm{~F}$ NMR $(376 \mathrm{MHz}$, 
$\left.\mathrm{CDCl}_{3}\right) \delta:-114.0(\mathrm{dt}, J=56.8,16.5 \mathrm{~Hz}, 2 \mathrm{~F})$; MS (EI) $\mathrm{m} / \mathrm{z}$ (\%): $194\left(\mathrm{M}^{+}\right), 129$ (100); HRMS calcd for $\mathrm{C}_{12} \mathrm{H}_{12} \mathrm{~F}_{2}$ 194.0907, found 194.0909.

Supporting Information Optimization of the reaction conditions, mechanistic studies and copies of ${ }^{1} \mathrm{H}$ NMR, ${ }^{19} \mathrm{~F}$ NMR and ${ }^{13} \mathrm{C}$ NMR spectra of compounds 3 and $\mathbf{6}$. The Supporting Information is available free of charge via the Internet at http://sioc-journal.cn.

\section{References}

[1] (a) Meanwell, N. A. J. Med. Chem. 2011, 54, 2529.

(b) Erickson, J. A.; McLoughlin, J. I. J. Org. Chem. 1995, 60, 1626.

[2] (a) Ge, S.; Chaladaj, W.; Hartwig, J. F. J. Am. Chem. Soc. 2014, $136,4149$.

(b) Gu, Y.; Leng, X.; Shen, Q. Nat. Commun. 2014, 5, 5405.

(c) Xu, L.; Vicic, D. A. J. Am. Chem. Soc. 2016, 138, 2536.

(d) Serizawa, H.; Ishii, K.; Aikawa, K.; Mikami, K. Org. Lett. 2016, 18,3686 .

[3] (a) Moore, G. G. I. J. Org. Chem. 1979, 44, 1708.

(b) Ruppert, I.; Schlich, K.; Volbach, W. Tetrahedron Lett. 1984, 25 , 2195.

(c) Tyutyunov, A. A.; Boyko, V. E.; Igoumnov, S. M. Fluorine Notes 2011, 74, 1.

(d) Prakash, G. K. S.; Hu, J.; Olah, G. A. J. Org. Chem. 2003, 68, 4457.
[4] Feng, Z.; Min, Q.-Q.; Zhang, X. Org. Lett. 2016, 18, 44.

[5] Feng, Z.; Min, Q.-Q.; Fu, X.-P.; An, L.; Zhang, X. Nat. Chem. 2017, 9, 918.

[6] Xu, C.; Guo, W.-H.; He, X.; Guo, Y.-L.; Zhang, X.-Y.; Zhang, X. Nat. Commun. 2018, 9, 1170 .

[7] Guiadeen, D.; Kothandaraman, S.; Yang, L.; Mills, S. G.; MacCoss, M. Terahedron Lett. 2008, 49, 6368.

[8] Fu, X.-P.; Xiao, Y.-L.; Zhang, X. Chin. J. Chem. 2018, 36, 143.

[9] Sheng, J.; Ni, H.-Q.; Bian, K.-J.; Li, Y.; Wang, Y.-N.; Wang, X.-S. Org. Chem. Front. 2018, 5, 606.

[10] (a) Feng, Z.; Xiao, Y.-L.; Zhang, X. Acc. Chem. Res. 2018, 51, 2264.

(b) Feng, Z.; Chen, F.; Zhang, X. Org. Lett. 2012, 14, 1938.

(c) Feng, Z.; Min, Q.-Q.; Xiao, Y.-L.; Zhang, B.; Zhang, X. Angew. Chem., Int. Ed. 2014, 53, 1669.

(d) Min, Q.-Q.; Yin, Z.; Feng, Z.; Guo, W.-H.; Zhang, X. J. Am. Chem. Soc. 2014, 136, 1230.

(e) Xiao, Y.-L.; Guo, W.-H.; He, G.-Z.; Pan, Q.; Zhang, X. Angew. Chem., Int. Ed. 2014, 53, 9909.

[11] Prinsell, M. R.; Everson, D. A.; Weix, D. J. Chem. Commun. 2010, 46, 5743.

[12] ${ }^{19} \mathrm{~F}$ NMR showed the chemical shifts of difluoromethyl Zinc species $\mathbf{A 1}$ and $\mathbf{A} 2$ are consistent with the literature, see: Burton, D. J.; Hartgraves, G. A. J. Fluorine Chem. 2007, 128, 1198.

[13] (a) Weix, D. Acc. Chem. Res. 2015, 48, 1767.

(b) Gu, J.; Wang, X.; Xue, W.; Gong, H. Org. Chem. Front. 2015, 3, 1411.

[14] Lu, C.-H,; Gu, Y.; Wu, J.; Gu, Y.-C.; Shen, Q.-L. Chem. Sci. 2017, 8,4848 . 